For reprint orders, please contact: reprints@futuremedicine.com

\title{
Antifungal prophylaxis: why, what and how?
}

Sue Lyon*

\section{1st Annual Meeting of the European Society for Blood and Marrow Transplantation (EBMT 2015), Istanbul, Turkey, 22-25 March 2015}

The European Society for Blood and Marrow Transplantation (EBMT) is the leading scientific society for professionals involved in hematopoietic stem-cell transplantation (HSCT) and represents 563 transplant centers from 57 countries within and outside Europe. Each year, the EBMT Annual Meeting brings together over 4,500 scientists, physicians, nurses, biologists, technicians and patients to discuss scientific data that build on past achievements in the field of HSCT. The procedure offers the chance of long-term remission of hematological and lymphoid cancers, but patients are at increased risk of serious infections, especially after allogeneic HSCT. These infections include the invasive fungal infections that were among the important topics discussed during EBMT 2015.

First draft submitted: 1 October 2015; Accepted for publication: 19 October 2015;

Published online: 17 December 2015

Hematopoietic stem-cell transplantation (HSCT) has made rapid progress since its introduction into clinical practice more than 50 years ago. Two years after the millionth transplant was reported, a record 25 million people worldwide are now listed as potential volunteer marrow donors. The announcement of this important milestone during the 41st Annual Meeting of the European Society for Blood and Marrow Transplantation (EBMT 2015) highlighted the exponential rise in allogeneic HSCT procedures, especially from unrelated donors, since the first successful unrelated transplant in 1973.

HSCT offers the chance of long-term remission for people with hematological and lymphoid cancers, but this success can come at a cost. Allogeneic HSCT recipients are at high risk of invasive fungal infections (IFI) due to prolonged and profound neutropenia or immunosuppression for graftversus host disease (GvHD). Despite the availability of antifungal treatment, mortality associated with IFI in hemato-oncology patients remains high at 30-50\% [1,2], with up three-quarters of cases diagnosed after death [3].

\section{Antifungal prophylaxis}

Persistent fever may be the only early sign of IFI, and the resulting diagnostic challenge, together with the high risk of mortality, have provided the rationale for antifungal prophylaxis in HSCT recipients. This also can apply to other high-risk hemato-oncology patients such as those with acute myeloid leukemia (AML) or myelodysplastic syndrome (MDS) undergoing intensive chemotherapy. According to an analysis of the Transplant-Associated Infection Surveillance Network

*Independent Medical Writer, London, UK; sue.lyon@writers-touch.com

\section{KEYWORDS}

- antifungal prophylaxis

- aspergillosis • candidemia

-EBMT • IFI • invasive fungal infections $\bullet$ invasive mold infections $\bullet$ hematopoietic stem-cell transplantation - HSCT

\section{Future : Medicine ${ }_{\text {part of }}$}


(TRANSNET) database, systemic antifungal prophylaxis with fluconazole has reduced the frequency of candidemia, but this has been associated with an increase in the incidence of invasive mold infections, especially aspergillosis [4].

This experience from the USA was reflected in a Spanish study presented during EBMT 2015 that included 403 pediatric patients who underwent HSCT between January 1991 and December 2013. Since triazole antifungal prophylaxis was introduced at the center, aspergillosis has become the most common IFI with an incidence of $2.7 \%$, most often presenting as pulmonary aspergillosis ( $75 \%$ of cases). In this study, overall mortality was $68.0 \%$ in patients with IFI, compared with $44.9 \%$ in the overall population [5].

Also presented during EBMT 2015, a second single-center Spanish study that included 252 consecutive allogeneic HSCT recipients investigated the effectiveness of antifungal prophylaxis following the introduction of voriconazole in 2004. Up to 2014, the incidence of IFI in patients receiving voriconazole as primary prophylaxis was $7.5 \%$. This represented a $50 \%$ reduction in incidence of IFI compared with incidence in a historical cohort of 98 patients who received fluconazole as antifungal prophylaxis between 2001 and 2004. In total, 81 patients discontinued voriconazole: 61 (24\%) temporarily and 20 $(8.3 \%)$ permanently. The commonest reason for discontinuation was adverse drug reactions (66 patients; $26.2 \%$ ), liver toxicity being the most frequent (44 patients, 17.4\%). Overall survival was $59 \%$ with a median follow up of 30 months (1-118 months) [6].

Since 2005, the European Conference in Infections in Leukaemia (ECIL) has produced evidence-based clinical guidelines designed to improve the management of patients at high risk of infectious complications due to the treatment of leukemia or following HSCT. The latest ECIL guidelines [7] recommend that prevention of infection in hemato-oncology patients should include: protection against construction or renovation activities as well as heavily contaminated items such as potted plants or soil; protective isolation with high-efficiency particular air (HEPA) filtration to prevent exposure; and mold-active prophylaxis to prevent disease [8].

Professor Rafael Duarte (Barcelona, Spain) reported that posaconazole is the only drug to be given two A1 recommendations by ECIL in antifungal prophylaxis: for AML patients undergoing intensive chemotherapy if baseline incidence of invasive mold infections is high, and for HSCT recipients with GvHD. Fluconazole receives an AI recommendation for antifungal prophylaxis in the pre-engraftment phase of HSCT when there is a low risk of invasive mold infections, but ECIL strongly recommends against using the drug during pre-engraftment when the risk of molds is high or in GvHD.

Voriconazole is also recommended for antifungal prophylaxis after allogeneic HSCT, but with a lower, BI recommendation. According to Professor Duarte, this grading is based on outcomes of two clinical trials that included allogeneic HSCT recipients. In a double-blind randomized study, there was a trend to fewer IFI with voriconazole than with fluconazole, but fungal-free survival, relapse-free and overall survival, and the incidence of severe adverse events were similar [9]. There was also no difference between voriconazole and itraconazole in the incidence of proven/probable IFI or survival to day 180 in a prospective, randomized, openlabel, multicenter study, though voriconazole could be given for significantly longer duration, with less need for other systemic antifungals [10].

Future options for antifungal prophylaxis may include isavuconazole, which has recently been approved in both Europe and the USA for the treatment of invasive aspergillosis and mucormycosis. Treatment with isavuconazole has been shown to be as efficacious as voriconazole - currently graded AI by ECIL for first-line treatment of invasive aspergillosis [7] - but it is significantly better tolerated, with fewer drug-related adverse events [11]. Isavuconazole was also safe and well tolerated when used prophylactically in an openlabel, dose escalation study in high-risk patients with AML [12]. Professor Duarte commented that isavuconazole's improved tolerability may make it a potentially attractive choice for antifungal prophylaxis if clinical data demonstrate its efficacy in this indication.

\section{New options for antimold prophylaxis}

Since posaconazole became a guideline-endorsed option for IFI prophylaxis, its use has increased in high-risk hemato-oncology patients, with evidence of a reduced incidence of breakthrough IFI compared with prophylaxis with itraconazole [13]. However, there have been concerns about the variability in serum levels found in patients receiving posaconazole oral suspension [14]. In addition, some patients find it 
difficult to tolerate the suspension, which must be administered with a full meal in multiple daily doses.

According to Professor Duarte, the recent introduction of a once-daily posaconazole oral tablet and an intravenous (iv.) formulation has helped to overcome these practical difficulties. He explained that both the EMA and the US FDA agreed that the clinical program for the new posaconazole formulations should involve a pharmacokinetic (PK) bridging strategy, which was designed to demonstrate comparable exposure and safety among the same patient populations for whom posaconazole oral suspension had already been approved. The target range for average serum concentration (Cavg) of $500-2500 \mathrm{ng} / \mathrm{ml}$ for $90 \%$ of subjects was chosen on the basis of the exposure-response relationship seen with posaconazole oral suspension in IFI prophylaxis and in studies conducted in refractory IFI $[15,16]$.

The once-daily oral tablet combines posaconazole with a $\mathrm{pH}$-dependent polymer that is designed to eliminate the need for food or multiple daily dosing to achieve systemic exposure. The PK and safety of the tablet were examined in a Phase III study that included 210 patients with acute myeloid leukemia or myelodysplastic syndrome and HSCT recipients. Of the 186 PK-evaluable patients, only one $(<1 \%)$ did not achieve the lower exposure target of $500 \mathrm{ng} / \mathrm{ml}$, and seven (4\%) exceeded the upper exposure target of $2500 \mathrm{ng} / \mathrm{ml}$. There were no unexpected treatment-related adverse events, the most commonly reported side effects being nausea $(11 \%)$ and diarrhoea (8\%). In a quartile analysis, there was no evidence of an increase in adverse events with higher posaconazole exposure [17].

Posaconazole iv. is an aqueous solution containing the solubilizer sulfobutyl ether betacyclodextrin - the same solubilizer that is used, and in a similar amount, in iv. voriconazole. Professor Duarte regarded the posaconazole iv. formulation as an especially useful therapeutic option in the intensive care unit, since critically ill patients may be unable to take or absorb oral medication. The Phase III PK and safety study for posaconazole iv. included 237 at-risk neutropenic patients with AML, MDS and post-allogeneic HSCT. Of the 49 PK-evaluable patients with $\geq 10$ days of iv. dosing, 46 (94\%) achieved steady-state Cavg 500-2500 ng/ml. Adverse events were similar to those previously reported for posaconazole oral suspension, the most common reported treatment-related adverse events being diarrhoea $(8 \%)$, nausea $(5 \%)$ and rash (5\%) [18].

\section{Prophylaxis in practice}

Randomized, double-blind clinical trials provide the gold-standard evidence base for choice of antifungal prophylaxis or treatment. However, Professor Dimitrios Kontoyiannis (TX, USA) reminded delegates that clinical trials may not reflect clinical practice, where there is often diagnostic uncertainty, and patients often have multiple comorbidities and co-infections, drug interactions and a history of previous antifungal treatment. It is, therefore, essential to also review clinical experience with any new therapy.

When the posaconazole oral tablet became available in the USA, nearly all patients receiving the oral suspension at Professor Kontoyiannis's center were 'bridged' to the oral tablet. A retrospective study of the center's pharmacy database found that posaconzole serum levels were lower with the suspension than with the tablet. When patients were treated with posaconazole suspension, only 12 of 21 (57\%) serum levels reached target levels for prophylaxis $(>700 \mathrm{ng} / \mathrm{ml})$ and only 5 of $21(24 \%)$ reached target levels for treatment $(>1000 \mathrm{ng} / \mathrm{ml})$. In contrast, after the patients had been switched to posaconazole tablets, target levels for prophylaxis and for treatment were achieved in respectively 29 of 30 (97\%) and in 25 of 30 (83\%) of serum levels [19].

Posaconazole tablets were generally well tolerated by Professor Kontoyiannis's patients. Seven days after switching from oral suspension to posaconazole tablets there were mild, asymptomatic increases in liver function tests (LFT) in five of the nine patients with normal baseline LFTs. Most (80\%) of these abnormalities were total bilirubin elevation. Liver enzymes returned to the normal range within 3 weeks, even though patients continued to receive posaconazole tablets. In two of the three patients with abnormal baseline LFT, liver enzyme levels fell after starting posaconazole tablets. Only one patient, who had severe GvHD (skin, liver, grade 3), experienced a sustained rise in LFTs, despite discontinuation of the tablets [19]. Professor Kontoyiannis concluded that studies with larger numbers of patients are needed to clarify any relationship between posaconazole serum level and clinically relevant hepatotoxicity or other adverse effects.

Other centers in the USA have reported that 
target posaconzole concentrations appear to be routinely available with posaconazole tablets compared with oral suspension [20]. However, one study found that subtherapeutic serum levels were more likely in patients with BMI $\geq 30$ or actual bodyweight $\geq 90 \mathrm{~kg}$ [21]. It, therefore, seems possible that a patient's weight and BMI may affect posaconazole serum levels, but again more studies are needed to confirm this observation.

\section{Conclusion}

Antifungal prophylaxis significantly reduces all-cause mortality compared with placebo or nonsystemic antifungals in allogeneic HSCT recipients and high-risk leukemia patients [22]. In Professor Kontoyiannis's experience, switching from posaconazole oral suspension to the oral tablet enables more patients to achieve therapeutic serum levels. Toxicity does not seem to be a problem after switching, but there may be a subset of heavier patients in whom standard dosing does not attain therapeutic serum levels. Consequently, the introduction of the posaconazole tablet has not eliminated the need for therapeutic drug monitoring, he concluded.

\section{Financial \& competing interests disclosure}

The attendance of $S$ Lyon at European Society for Blood and Marrow Transplantation and her contribution to the writing of this report were supported by Merck Sharp and Dohme. The author has no other relevant affiliations or financial involvement with any organization or entity with a financial interest in or financial conflict with the subject matter or materials discussed in the manuscript apart from those disclosed.

No writing assistance was utilized in the production of this manuscript.

\section{References}

1 Pagano L, Caira M, Nosari A et al. Fungal infections in recipients of hematopoietic stem cell transplants: results of the SEIFEM B-2004 Study - sorveglianza epidemiologica infezioni fungine nelle emopatie maligne. Clin Infect Dis. 45, 1161-1170 (2007).

2 Pagano L, Caira M, Candoni A et al. Invasive aspergillosis in patients with acute myeloid leukemia: a SEIFEM-2008 registry study. Haematologica 95, 644-650 (2010).

3 Chamilos G, Luna M, Lewis RE et al. Invasive fungal infections in patients with hematologic malignancies in a tertiary care cancer center: an autopsy study over a 15 -year period (1989-2003). Haematologica 91, 986-989 (2006).

4 Kontoyiannis DP, Marr KA, Park BJ et al. Prospective surveillance for invasive fungal infections in hematopoietic stem cell transplant recipients, 2001-2006: overview of the Transplant-Associated Infection Surveillance Network (TRANSNET) database. Clin. Infect. Dis. 59, 1091-1100 (2010).

5 Vega HDR, Scocorro CF, Guerola BT, Navarro JMF. Invasive fungal infection in pediatric hematopoietic stem cell transplantation, Abstract P268. Presented at: 41st Annual Meeting of the European Society for Blood and Marrow (EBMT). Istanbul, Turkey, 22-25 March 2015.

6 Acevedo CC, Poyato MJL, Torres EG et al. Voriconazole as antifungal prophylaxis in allogeneic hematopoietic stem cell transplantation: ten years of experience in a single institution, Abstract PO25. Presented at: 41st Annual Meeting of the European Society for Blood and Marrow Transplantation. Istanbul, Turkey, 22-25 March 2015.

7 Herbrecht R. 2013 - update of the ECIL guidelines for antifungal therapy in leukaemia and HSCT patients (ECIL-5). Presented at: European Conference on Infections in Leukaemia (ECIL). Sophia Antipolis, France, 19-21 September 2013. www.kobe.fr/ecil/telechargements2013/ ECIL5\%20Antifungal\%20Therapy.pdf

8 Maertens J. Primary antifungal prophylaxis. Presented at: European Conference on Infections in Leukaemia (ECIL). Sophia Antipolis, France, 19-21 September 2013. www.kobe.fr/ecil/telechargements2013/ ECIL5antifungalprophylaxis\%20 20062014Final.pdf

9 Wingard JR, Carter SL, Walsh TJ et al. Randomized, double-blind trial of fluconazole versus voriconazole for prevention of invasive fungal infection after allogeneic hematopoietic cell transplantation. Blood 116, 5111-5118 (2010).

10 Marks DI, Pagluica A, Kibbler CC et al. Voriconazole versus itraconazole for antifungal prophylaxis following allogeneic haematopoietic stem-cell transplantation. $\mathrm{Br}$. J. Haematol. 155, 318-327 (2011).

11 Kontoyiannis D, Giladi M, Lee M et al. A Phase 3, randomized, double-blind, non-inferiority trial to evaluate efficacy and safety of isavuconazole versus voriconazole in patients with invasive mold disease
(SECURE): outcomes in invasive aspergillosis patients, Abstract 1211. Presented at: Infectious Diseases Week. Philadelphia, PA, USA, 8-12 October 2014.

12 Cornely OA, Bohme A, Hoffman AS, Ullmann AJ. Safety and pharmacokinetics of isavuconazole as antifungal prophylaxis in acute myeloid leukemia patients with neutropenia: results of a Phase 2, dose escalation study. Antimicrob. Agents Chemother. 59, 2078-2085 (2015).

13 Pagano L, Caira M, Candoni A et al. Evaluation of the practice of antifungal prophylaxis use in patients with newly diagnosed acute myeloid leukemia: results from the SEIFEM 2010-B registry. Clin. Infect. Dis. 55, 1515-1521 (2012).

14 Gross BN, Ihorst G, Jung M et al. Posaconazole therapeutic drug monitoring in the real-life setting: a single-center experience and review of the literature. Pharmacother. 10, 1117-1125 (2013).

15 European Medicines Agency Committee for Medicinal Products for Human Use (CHMP). Assessment report: Noxafil (international non-proprietary name: posaconazole). EMA/159150/201420 February 2014.

16 FDA. Clinical review. Posaconazole delayed release tablets. 6 March 2009.

17 Cornely OA, Duarte RF, Haider S et al. Phase 3 pharmacokinetics (PK) and safety study of posaconazole (POS) tablet in patients at risk for invasive fungal infection. Presented at: European Congress of Clinical Microbiology and Infectious Diseases (ECCMID). Berlin, Germany, 27-30 April 2013. 
Cornely OA, Haider S, Grigg A et al. Phase 3 pharmacokinetics (PK) and safety study of posaconazole (POS) IV in patients (Pts) at risk for invasive fungal infection (IFI), Abstract A-292. Presented at: 54 th Interscience Conference on Antimicrobial Agents and Chemotherapy (ICAAC). Washington DC, USA, 5-9 September 2013.

19 Jung DS, Tverdek FP, Kontoyiannis DP. Switching from posaconazole suspension to tablets increases serum drug levels in leukemia patients without clinically relevant hepatotoxicity. Antimicrob. Agents Chemother. 58, 6993-6995 (2014).

20 Jancel T, Kumar P, Malech HL et al. Posaconazole serum concentrations of the delayed-release tablets compared with the oral suspension, Abstract A-703. Presented at: 54th Interscience Conference on Antimicrobial Agents and Chemotherapy (ICAAC).

Washington DC, USA, 5-9 September 2014.

21 Miceli MH, Perissinotti AJ, Kaufman C et al. Clinical experience using posaconazole (pos) extended release tablet (tab) for the prevention of invasive fungal infections in haematological cancer patients (pt): does one size fit all? Abstract M-1759. Presented at: 54th Interscience Conference on Antimicrobial Agents and Chemotherapy (ICAAC). Washington DC, USA, 5-9 September 2014.

22 Robenshtok E, Gafter-Gvili A, Goldberg E et al. Antifungal prophylaxis in cancer patients after chemotherapy or hematopoietic stem-cell transplantation: systematic review and metaanalysis. J. Clin. Oncol. 25, 5471-5489 (2007) 